Military Technical College, Kobry El-Kobbah, Cairo, Egypt

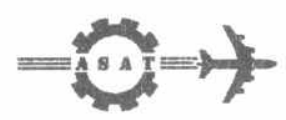

$9^{\text {th }}$ International Conference

On Aerospace Sciences \&

Aviation Technology

\title{
AN UNSUPERVISED NEURAL NETWORK FOR IMPULSIVE NOISE REMOVAL IN DIGITAL IMAGES
}

\author{
FAYED* K.A. and MAHMOUD** T.A.
}

\begin{abstract}
Pictures or images play an important role as a mass communication medium. When images are coded and transmitted over noisy communication channels, images are often corrupted by impulse noise. A method is proposed to eliminate impulsive noise with gaussian or uniform distribution in digital images. This noise removing method is based on two steps: impulse noise detection and filtered image reconstruction. Motivated by the success of neural computing in pattern classification, an unsupervised neural network has been employed in detecting the positions of the noisy pixels. When the noisy pixels are detected, a number of noise-exclusive filtering algorithms are invoked to eliminate the noise. These filters do not affect those pixels that are not corrupted. The filtering scheme presented can suppress impulse noise effectively as well as avoiding blurring or degrading the digital image quality. Experimental results and associated statistics demonstrate that the performance of the noise-exclusive filters is superior to many other well-known methods
\end{abstract}

\section{KEYWORDS}

Impulse noise removal, Unsupervised neural network, Noise-exclusive filtering, Digital image processing.

*Ph. D., Egyptian Armed Forces.

*\#. Sc., Egyptian Armed Forces. 


\section{INTRODUCTION}

Human vision is one of the most important and complex perception mechanisms. It provides information needed for relatively simple tasks like object recognition as well as very complex tasks such as planning, decision making and scientific research. Digital image processing has exhibited an enormous growth and created an important technological impact in several areas, such as in telecommunications, TV broadcasting as well as medicine and scientific research [1]

Noise refers to a variety of undesirable disturbances owing to errors of all types and interference from external sources. Impulse noise usually appears during image and TV picture transmissions [2]. It is impulsive noise and its source is either atmospheric or man-made. Impulse noise is also called "salt-pepper" noise. There are two models for impulsive noise [3]. In the first model all noise pixels get one of the two fixed values. This kind of impulse noise has been successfully eliminated by a method given in [4]. The second model enables the intensity of the impulses to follow random distributions. This model can be expressed mathematically as:

$$
X_{i}= \begin{cases}I_{p}+N_{r} & \text { with probability } P_{p} \\ I_{n}+N_{r} & \text { with probability } P_{n} \\ E_{i} & \text { with probability } 1-\left(P_{p}+P_{n}\right)\end{cases}
$$

Where:
$X_{i}$ denotes the pixel values of the degraded image.
$I_{p}, I_{n}$ denote the mean values for positive and negative impulse noise.
$\mathrm{N}_{\mathrm{r}} \quad$ has a zero-mean and may follow a gaussian (or uniform)
$E_{i} \quad$ distribution and standard deviation $\sigma$.

Image enhancement process is comprised of a collection of techniques that seek to improve the visual appearance of an image, or to convert the image to a form more suitable to human or machine analysis. Median filters have some very interesting properties. They have low-pass characteristics and they remove additive white noise. Since the median is a robust estimator of location, it is very suitable for impulse noise filtering. The principal function of the median filter is to force points with distinct intensities to be more like their neighbors. The median filter not only smoothes noise in homogeneous image regions but tends to produce regions of constant or nearly constant intensity as well. Thus, it removes very fine details and changes signal structure. Moreover, the median filter becomes unreliable only if more than $50 \%$ of the data are corrupted [5]. To improve the performance of the median filter, many generalized median filters such as the center weighted median (CWM) filter [6], the maximum/median (max/med) filter and the multistage median filter have been proposed [7]. The generalized median filters tend to have better detail preserving 
characteristics than the median filter but they preserve more details at the expense of poor noise suppression.

In this paper, an approach for effective suppression of impulsive noise following model (1) from images while preserving image integrity is proposed. The scheme presented is based upon impulsive noise detection. If the impulses can be detected and their positions are correctly located in the image, then it is feasible to replace the corrupted pixels by the best estimates using only the uncorrupted pixels.

\section{IMPULSE NOISE DETECTION}

\subsection{Image Segmentation by an Unsupervised Learning Algorithm}

The automatic segmentation of images is an important component of visual information processing. The objective of segmentation is to classify a given image into meaningful regions that are homogeneous according to certain properties, such as gray level, texture or color [8]

The use of neural networks in pattern classification is a very popular issue in the study of neural computing. Two basic categories of classification methods exist for machine learning: supervised and unsupervised. Comparing with classical segmentation techniques, the unsupervised (self-organizing) neural network has the following advantage [9]:

(a) It does not require prior information, not even the number of classes. It completes the identification of patterns and classification all by its autonomous learning

(b) Segmentation by the self-organizing neural network is completed by one pass without any preprocessing manipulation.

The proposed neural network in this paper has a structure similar to that of ART (Adaptive Resonance Theory). This net clusters input patterns by using unsupervised learning. It has two layers: the input layer and the output layer. Feed-forward paths connect these layers. The nodes of the output layer are lateral inhibitive to one another. They are designed as a competitive network capable of choosing the winning node. Each time a pattern is presented, an appropriate cluster unit is chosen and the cluster's weights are adjusted to let the cluster unit learn the pattern. As is often the case in clustering nets, the weights on a cluster unit may be considered to be a model for the patterns placed on that cluster [10].

\subsection{Feature Selection}

Noisy pixels can be characterized by their local statistical properties. To extract features from local statistics, a window is used to pass through the entire noise degraded image. The size of the window $W_{D}$ is arbitrary. However, a $3^{*} 3$ window is good enough in most applications. From the small window, useful local features are obtained such as mean, median, variance, range, etc. 
Assume that features are measured from each input pattern. Each set of these features is considered as a vector in the feature space. The problem of classification is to assign each possible vector to a proper pattern class

Among the previously mentioned local features, two local features are chosen to form the input vector $Z$. One is the pixel value and the other is the median deviation that is calculated from the difference between the median of the pixels in the window and the pixel value [11]. Although the median deviation is easy to operate and is effective when the image is slightly contaminated with impulse noise. However, it can not perform well when the noise ratio - which is the ratio between the corrupted pixels and the uncorrupted pixels - is high. To further improve the detection accuracy, the pixel value is also introduced as an input feature [12][13].

Thus:

$$
Z=\left(Y_{1}, Y_{2}\right)
$$

Where:

$$
\begin{aligned}
& Y_{1}=X_{1} \\
& X_{i} \text { is the value of the pixel in the center of the window. } \\
& Y_{2}=\underset{g}{\operatorname{median}}\left(X_{1-g}, \ldots, X_{1}, \ldots, X_{i+g}\right)-X_{i}
\end{aligned}
$$

The distribution of the input vectors is shown in Fig. 1. The vertical axis denotes the pixel value and the horizontal axis denotes the distribution of the median deviation. Positive impulse noise coalesces at the top left of the figure; signals are shown as vertical bar in the center; and negative impulse noise is at the right bottom.

\subsection{Unsupervised Learning Algorithm}

The essential point of this algorithm is to build up the clusters using the Euclidean distance measure betvreen the input $Z$ and the weights $U$, assuming:

$$
U_{i}=\left(U_{i 1}, U_{i 2}\right)
$$

The algorithm is summarized as follows [4][11]

Step 1:

Initialize the weight vectors with random values (The first sample is simply taken as tha representative of the first cluster).

Step 2 :

Presen't a new sample to the input layer of the network, and compute the Euclidrean distance $D_{i}$ between the sample and all the weight vectors using: 


$$
D_{i}=\sqrt{\sum_{j=1}^{2}\left(Z_{j}(t)-U_{i j}(t)\right)^{2}} \quad(i=1, \ldots, q)
$$

Where $\mathrm{q}$ is the number of clusters

Step 3:

Select the winning node $i^{*}$ with minimum $D$.

$$
\begin{aligned}
& D_{i}=\min \left\{D_{1}\right\} \\
& \text { If } D_{i} \leq T_{1} \quad I^{*} \text { Where } T_{1} \text { is the predefined threshold } * /
\end{aligned}
$$

Then

Assign $Z$ to the $i^{\text {th }}$ cluster and update the weight vector $U_{1}$ according to the following learning rule:

$U_{i}(t+1)=U_{i}(t)+\alpha\left[Z(t)-U_{i}(t)\right]$

Where: $\alpha$ is the learning rate; $0<\alpha<1$.

$\alpha=\left\{\alpha_{m}, \alpha_{p}\right\}$

$\alpha_{m}$ is the learning rate for the median deviation.

$\alpha_{p}$ is the learning rate for the pixel value.

In order to define the values of $\alpha_{m}$ and $\alpha_{p}, F_{i}$ is calculated. $F_{i}{ }^{i}$ is the difference in the pixel values between the pixel value of the winning node and that of the sample.

If $\quad \mathrm{F}_{\mathrm{i}} \leq \mathrm{T}_{2} \quad / *$ Where $\mathrm{T}_{2}$ is the predefined threshold */

Then

A very small value is given to the learning rate $\alpha_{p}$ to update the weight vector of the pixel values and a value in the range of 0.5 to $\alpha_{\mathrm{m}}$ to change the weight vector of the median deviations.

\section{Else}

Both $\alpha_{p}$ and $\alpha_{m}$ are set to 0.5 in order to update the weight vector of both the pixel values and that of the median deviations.

Else

Form a new cluster starting with $Z$.

Step 4:

Repeat by going back to step 2 until the input vector $Z$ is finished.

Fig. 2(a) and Fig. 2(b) describe the implementation of the above algorithm. As for Fig. 3 , it illustrates the architecture of the proposed neural network. 
The pixel values and the median deviations are then used to identify the noise classes. Sorting of $Z$ is first performed in terms of median deviation. A number of pixels values corresponding to the maximum and the minimum median deviations are selected. The histogram of these values is calculated, and the peak value of each group is selected as the noise class.

\section{IMPULSE NOISE REMOVAL}

Since the cluster centers, which represent the impulse noise, have been detected, the recovery of image becomes the process of matching pixels with the cluster centers.

Several noise-exclusive filters are introduced: the noise-exclusive arithmetic mean (NEAM) filter, the noise-exclusive median (NEM) filter, the noise-exclusive neighbors (NEN) filter and the noise-exclusive mean-median (NEMM) hybrid filter. These filters are used to restore the impulse corrupted images. The window size of these filters $W_{F}$ may vary as does the traditional median-type filters. The noise-exclusive filters mean that all the impulses in the window do not participate in the operation of order sorting in the median calculation or do not count in the operation of the mean calculation. This is fundamentally different from the conventional median and mean filtering where all noisy pixels inside the window $W_{F}$ are involved in the calculation.

As the noisy pixels are detected, the noise-exclusive filters are only invoked to eliminate them without affecting those pixels that are not corrupted. The NEAM filter and the NEM filter replace the impulses with the mean value and the median of the uncorrupted pixels respectively. The NEN filter replaces the noisy pixels with the nearest uncorrupted neighbor pixel. The NEMM replaces the impulses with the mean value of the uncorrupted pixels in the window $W_{F}$ and then takes the value of the median. Since the estimations are based on the uncorrupted pixels, the best possible restoration can be achieved [4][11] and [12].

\section{EXPERIMENTAL RESULTS AND ERROR ANALYSIS}

The performance of the proposed adaptive filters and that of the traditional median filter family have been further compared and tested on simulated examples. Error measurements are used to fairly compare the performance of each filter. The types used are the normalized mean square error (NMSE) and the projection mean square error (PMSE).

The NMSE is used to give a quantitative evaluation on the filtering results. The NMSE is calculated from the following formula: 


$$
\text { NMSE }=\frac{\sum_{i=-N / 2}^{N / 2} j=\frac{\sum_{i=-N / 2}^{N / 2}\left(S_{i j}-Y_{i j}\right)^{2}}{\sum_{N / 2}^{N / 2}} \sum_{j=-N / 2}^{N / 2}\left(S_{i j}\right)^{2}}{\sum^{N} / 2}
$$

Where $S_{i j}$ is the original image, $Y_{i j}$ is the filtered image and $N^{\star} N$ is the size of the image.

The PMSE is the mean square error projected along any image axis. The PMSE is used to study the characteristic of the error overall the filtered image and is described mathematically by:

$$
\operatorname{PMSE}(i)=\frac{\sum_{j=-N / 2}^{N / 2}\left(S_{i j}-Y_{i j}\right)^{2}}{\sum_{i=-N / 2}^{N / 2} \sum_{j=-N / 2}^{N / 2}\left(S_{i j}\right)^{2}}
$$

In this paper, two examples are illustrated to discuss the performance of the filters. Fig. 4(a) shows "Lenna" image $256 \times 256$ size. In the first example, "Lenna" image is corrupted by $40 \%$ positive and negative impulse noise, as shown in Fig. 4 (b). In this image, $N_{r}$ follows a uniform distribution with standard deviation $\sigma=20$. In the second example, "Lenna" image is corrupted by $60 \%$ positive and negative impulse noise, as shown in Fig. 8. In this image, $\mathrm{N}$, follows a gaussian distribution with standard deviation $\sigma=10$. The images processed by the median filter, the CWM filter, the max/med filter and the multistage median filter for the image shown in Fig. 4(b) are given in figures $5(\mathrm{a}), 5(\mathrm{~b}), 5(\mathrm{c})$ and $5(\mathrm{~d})$ respectively. While the images processed by the above mentioned filters for the image shown in Fig. 8 are given in figures 9(a), 9(b), 9(c) and 9(d) respectively. Fig. 6(a) and Fig. 10(a) show the distribution of the input vector $\left(Y_{1}, Y_{2}\right)$ fed to the neural network for images in Fig. $4(\mathrm{~b})$ and Fig. 8 respectively. Fig. $6(\mathrm{~b})$ and Fig. 10(b) show the clusters classification done by the neural network for images in Fig. 4(b) and Fig. 8 respectively. In figures 7(a), 7(b), $7(\mathrm{c})$ and $7(\mathrm{~d})$, the proposed NEAM, NEM, NEN, and NEMM process the image shown in Fig. 4(b) respectively. While the images processed by the above mentioned filters for the image shown in Fig. 8 are given in figures 11 (a), 11(b), 11(c) and 11(d) respectively.

The NMSE's of the "Lenna" image are calculated for different percentages of impulse noise. Fig. 12 shows the NMSE's calculated for each filter (F) to "Lenna" image corrupted by different percentages of impulse noise (\%I) with uniform. distribution with $\sigma=20$. Fig. 13 shows the same result but for impulse noise with gaussian distribution with $\sigma=10$.

The PMSE's are calculated for the filtered image of "Lenna". Fig. 14 shows the PMSE calculated for the NEAM filter, as a sample of the noise-exclusive median filters and the median filter, as a sample of the traditional median-type filters, applied 
on the "Lenna" image corrupted by $40 \%$ impulse noise with uniform distribution with $\sigma=20$. Similarly, Fig. 15 shows the PMSE calculated for the NEAM filter, as a sample of the noise-exclusive median filters and the CWM filter, as a sample of the traditional median-type filters, applied on the "Lenna" image corrupted by $60 \%$ impulse noise with gaussian distribution with $\sigma=10$.

From the figures of the filtered images, the tables and curves of NMSE of different percentages of impulse noise following gaussian and uniform distribution some conclusions are reached. Comparison of the filtered images clearly indicates that the noise-exclusive filters outperform the traditional median ones. The NMSE tables and curves demonstrate that the noise-exclusive filters achieve the smallest NMSE in all cases. Thus, the adaptive filters are better than the traditional median-type filters especially when impulse noise ratios are high (more than $30 \%$ impulse noise). From the PMSE figures, it is evident that the noise-exclusive filters have a lower PMSE and consistent overall the images as well, while the traditional median-type filters have higher PMSE all over the image especially at the edges, moreover they have larger fluctuations along the image axis.

\section{CONCLUSIONS}

The neural network guided adaptive filters have the ability to remove impulse noise in images. This has been done by detecting the positions of the noisy pixels and then applying a number of noise-exclusive filters. By utilizing the uncorrupted image pixels only, the scheme is capable of effectively eliminating the impulses with gaussian or uniform distribution while retaining image integrity. Experimental results and associated statistics have indicated that the proposed algorithm provides major improvement over many other well-known median-type filters in the aspects of noise removal, edge and fine detail preservation, as well as minimal signal distortion.

However, the traditional median-type filters have smaller time calculations than the proposed method. Therefore, the proposed filters have been used for better noise removal and minimal signal distortion especially at high percentages of impulse noise regardless of the processing time. On the other hand, the traditional median-type filters with a small processing time can be used especially at low percentages of impulse noise at the expense of minimum noise removal and signal distortion occurrence.

\section{REFERENCES}

[1] I. Pitas, Digital Image Processing Algorithms, Prentice Hall, 1993.

[2] Q. Zhang, R. Ward, "Impulse Noise Correction in TV Transmission", Proc. IEEE Consumer Electronics Conference, pp.292-293, 1995.

[3] B. Justusson, Median Filtering: Statistical Properties, in Two-Dimensional Digital Signal Processing II, Springer-Verlag, 1981. 
[4] K. Fayed, S. Shouman, S. Alian, T. Mahmoud, "Detection and Removal of Impulse Noise in Digital Images", Proc. of the $2^{\text {nd }}$ International Conference on Electrical Engineering, pp.707-715, Kobry Elkobbah, Cairo, Egypt, November 1999.

[5] W. Pratt, Digital Image Processing, John Wiley and Sons, 1978

[6] S. Ko, Y. Lee, "Center Weighted Median Filters and Their Applications to Image Enhancement", IEEE Transactions on Circuits and Systems, Vol.38, No.9, pp.984-993, September 1991.

[7] G. Arce, R. Foster, "Detail-Preserving Ranked-Order Based Filters for Image Processing", IEEE Transactions on Acoustics, Speech, and Signal Processing, Vol. 37, No.1, pp.83-98, January 1989.

[8] D. Ballard, C. Brown, Computer Vision, Prentice Hall, 1982.

[9] H. Kong, L. Guan, "A Self-Organizing Neural Network for Image Segmentation", Proc. $2{ }^{\text {nd }}$ Australian and New Zealand Conference on Intelligent Information Systems, pp.27-31, Brisbane, Australia, December 1994

[10] G. Carpenter, S. Grossberg, "The ART of Adaptive Pattern Recognition by a Self-Organizing Neural Network", IEEE Computer, pp.77-88, March 1988.

[11] H. Kong. L. Guan, "An Adaptive Approach for Removing Impulsive Noise in Digital Images", Proc. IEEE International Conference on Acoustics, Speech, and Signal Processing, Vol.4, pp.2287-2290, Piscataway, NJ, USA, 1996.

[12] Z. Wang, D. Zhang, "Progressive Switching Median Filter for the Removal of Impulse Noise from Highly Corrupted Images", IEEE Transactions on Circuits and Systems-II: Analog and Digital Signal Processing, Vol.46, No.1, pp.78-80, January 1999.

[13] Z. Wang, D. Zhang, "Restoration of Impulse Noise Corrupted Images Using Long-Range Correlation" IEEE Signal Processing Letters, Vol.5, No.1, pp 4-7, January 1998.

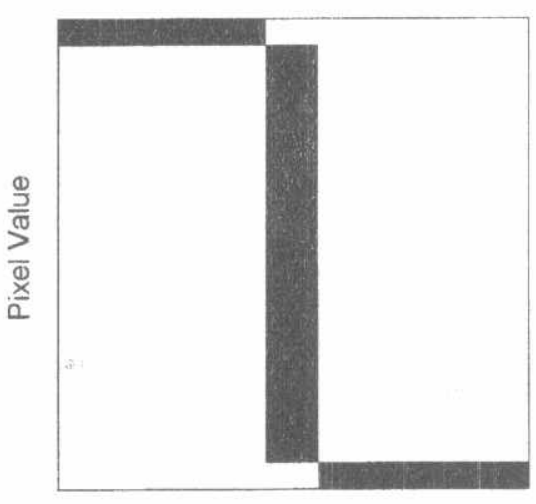

Median Deviation

Fig. 1 Typical 2-D distribution of the input vectors 


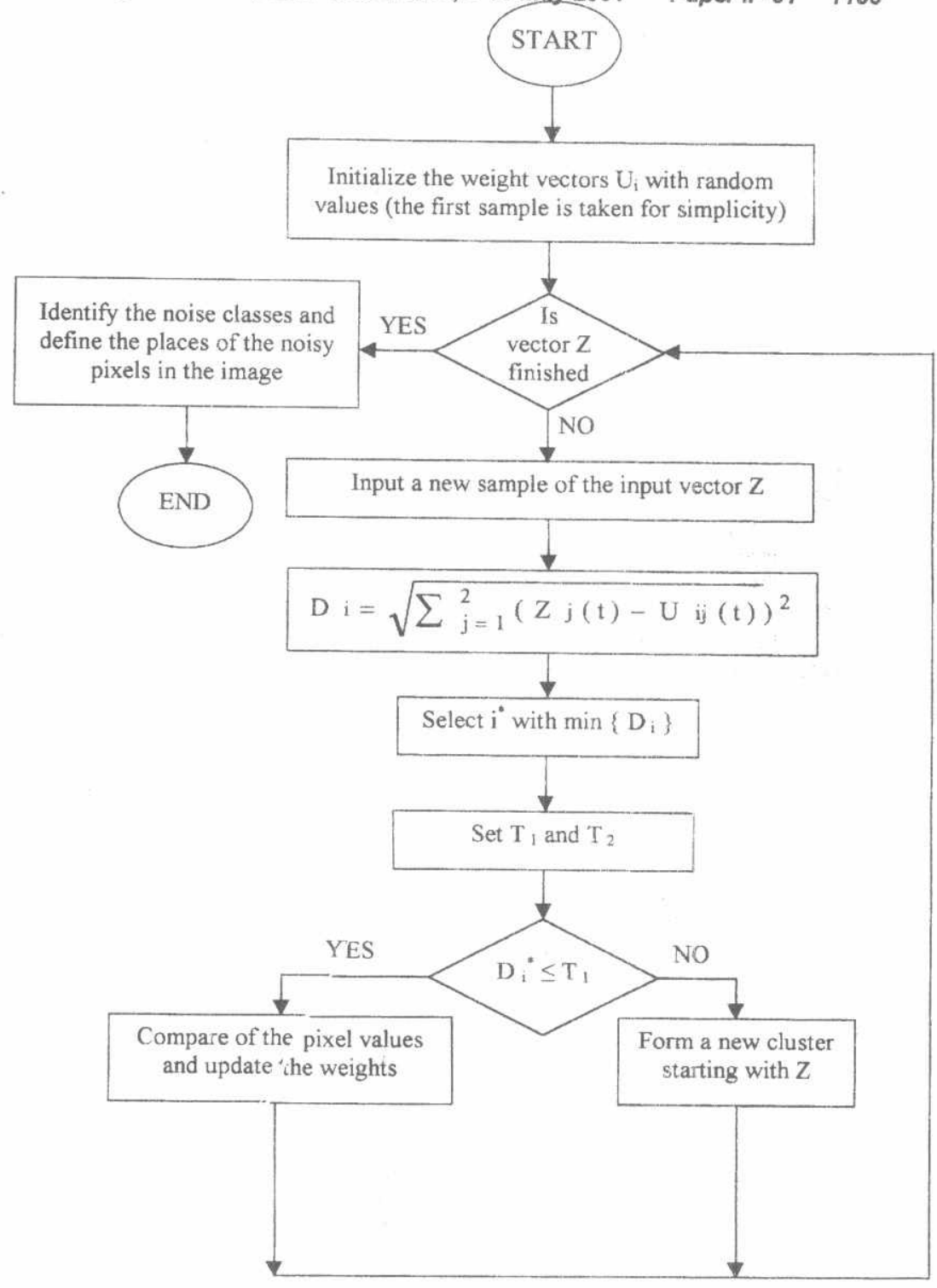

Fig. 2(a) Flow chart of unsupervised learning subroutine 


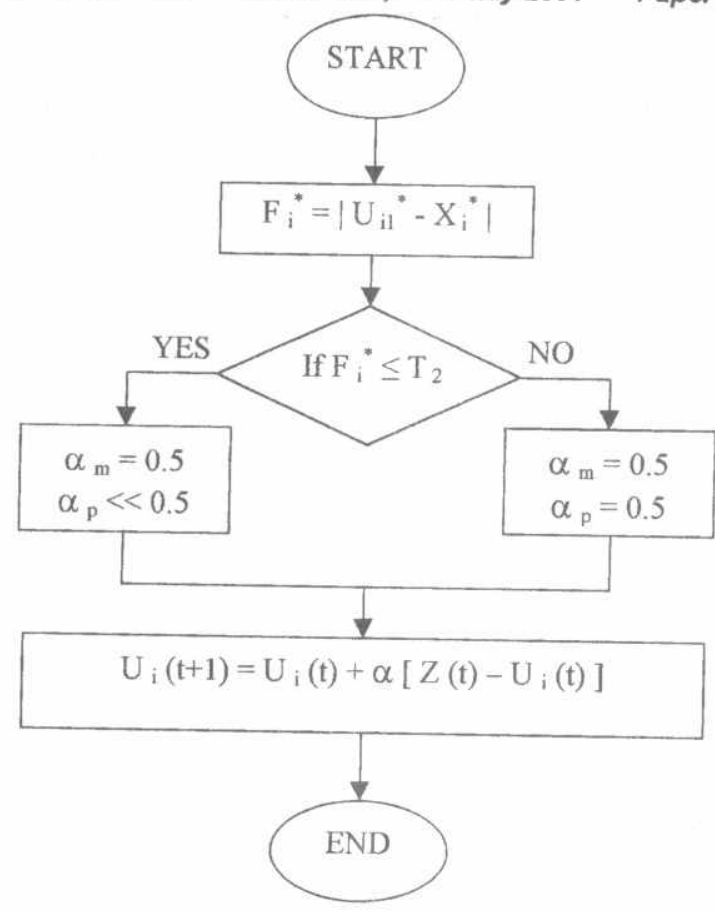

Fig. 2(b) Comparison of the pixel values and update the weights subroutine

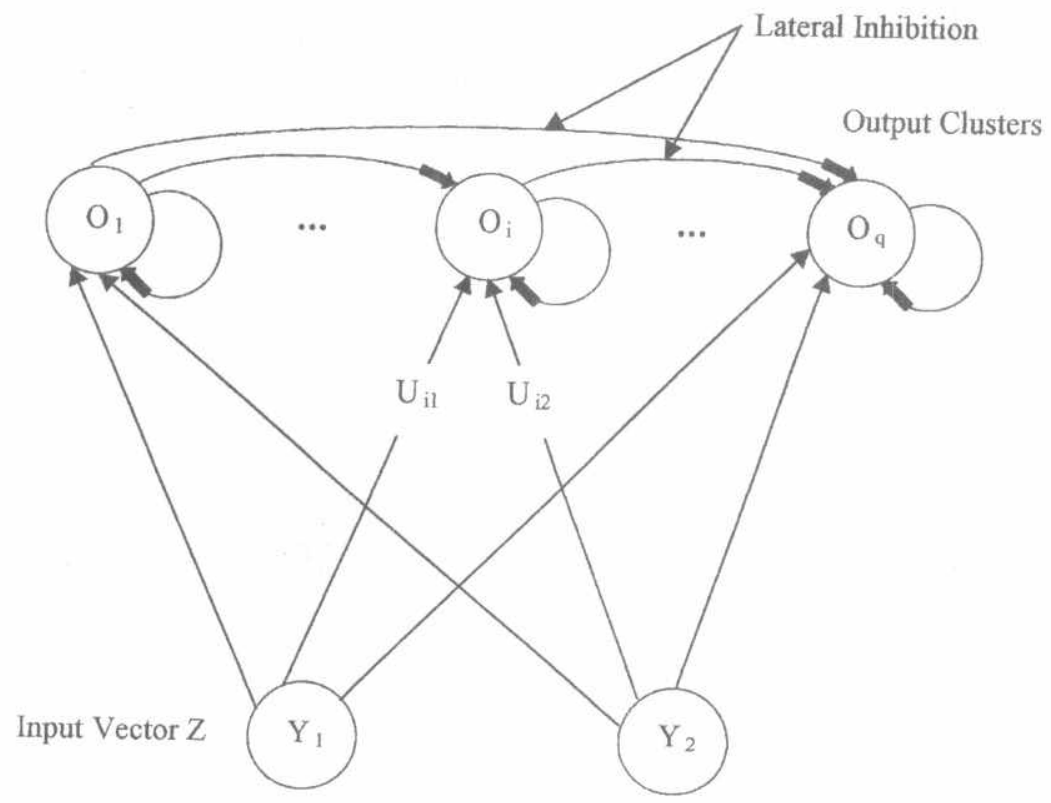

Fig. 3 Schematic representation of the used neural network 


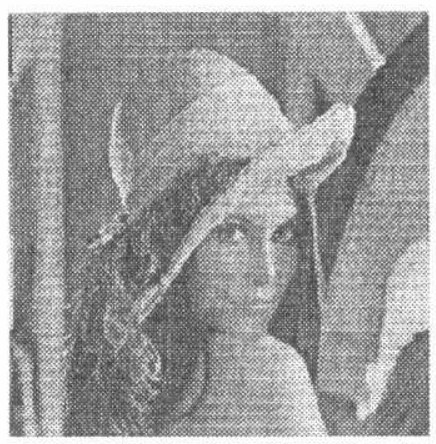

Fig. 4(a) Lenna image

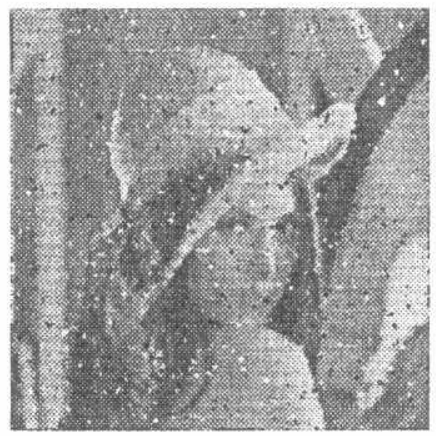

Fig. 5(a) Median filtered

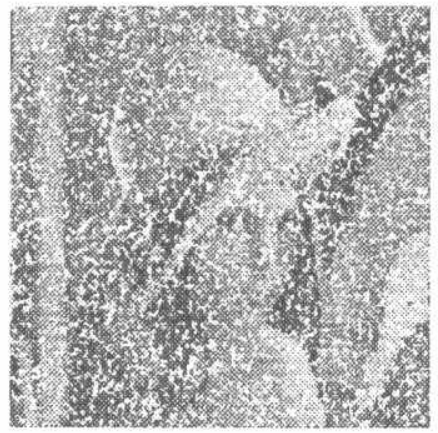

Fig. 5(c) Max/Med filtered

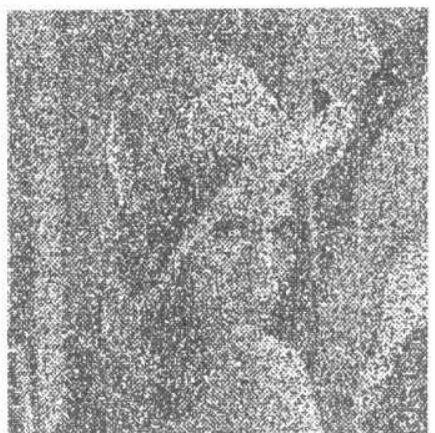

Fig. 4(b) Lenna image corrupted by $40 \%$ impulse noise with uniform distribution with $\sigma=20$

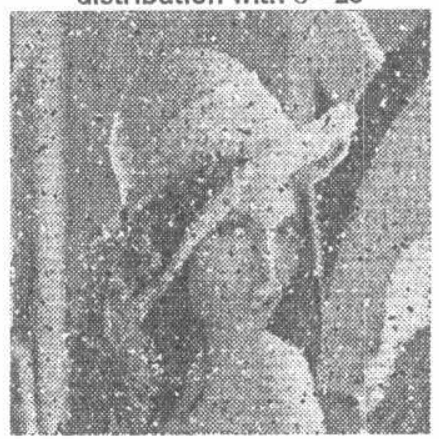

Fig. 5(b) CWM filtered

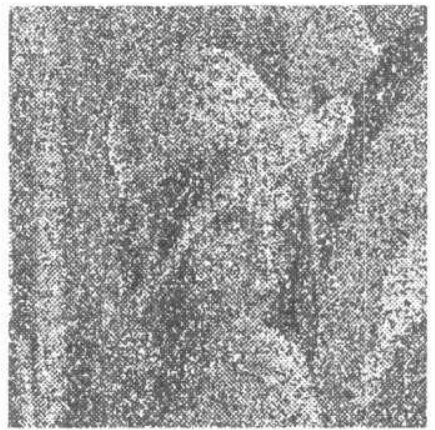

Fig. 5(d) Multistage filtered

Fig. 5 Traditional filtered images of Lenna image corrupted by $40 \%$ impulse noise with uniform distribution with $\sigma=20$ 


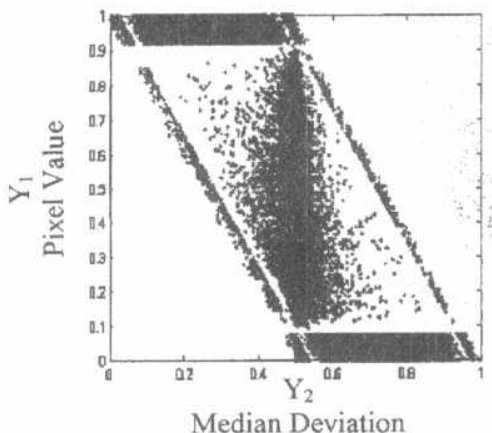

Fig. 6(a) Input vectors

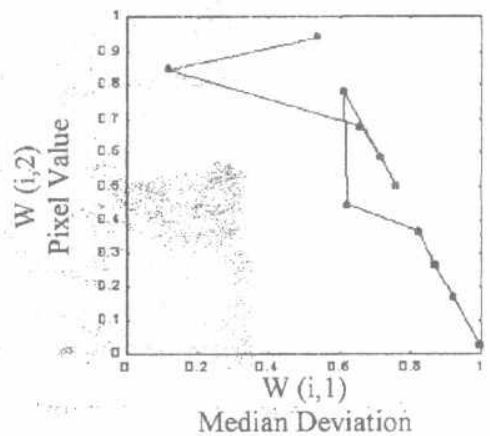

Fig. 6(b) Output clusters

Fig. 6 Noisy Lenna input vectors and output clusters of ANN applied to Lenna image corrupted by $40 \%$ impulse noise with uniform distribution with $\sigma=20$

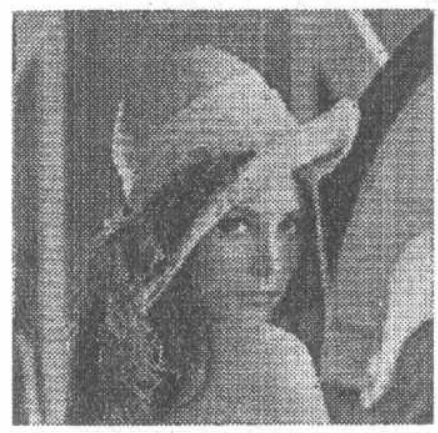

Fig. 7(a) NEAM filtered

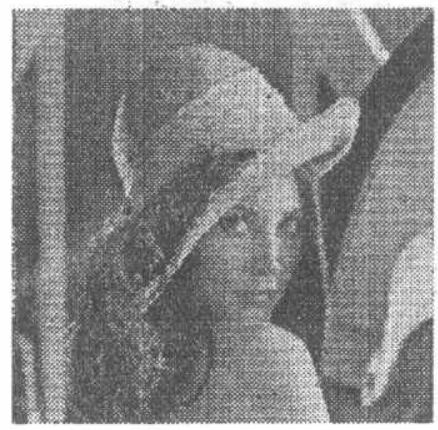

Fig. 7 (c) NEN filtered

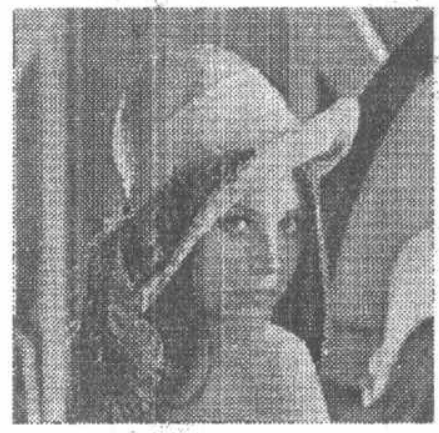

Fig. 7 (b) NEM filtered

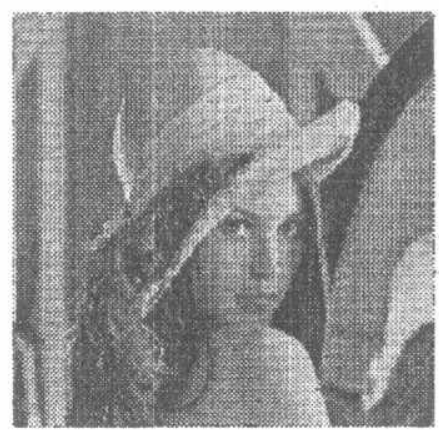

Fig. 7 (d) NEMM filtered

Fig. 7 Noise-exclusive filtered images of Lenna image corrupted by $40 \%$ impulse noise with uniform distribution with $\sigma=20$ 


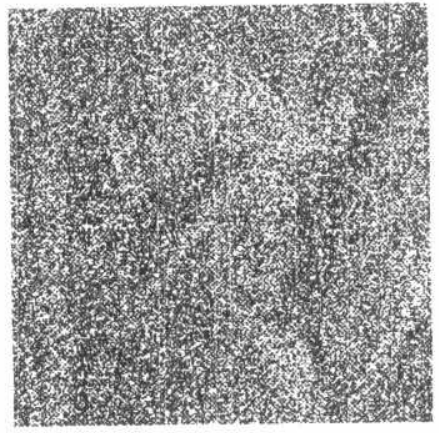

Fig. 8 Lenna image corrupted by $60 \%$ impulse noise with gaussian distribution with $\sigma=10$

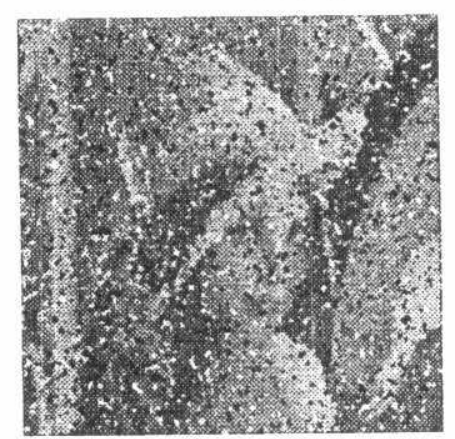

Fig. 9(a) Median filtered

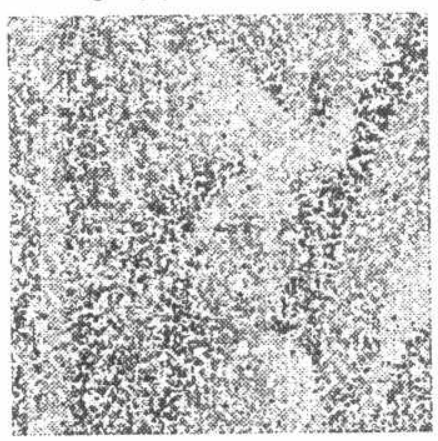

Fig. 9(c) Max/Med filtered

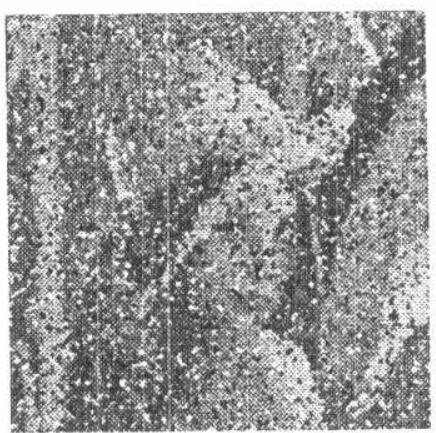

Fig. 9(b) CWM filtered

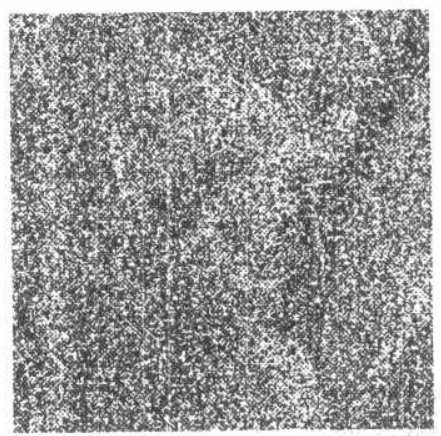

Fig. 9(d) Multistage filtered

Fig. 9 Traditional filtered images of Lenna image corrupted by $60 \%$ impulse noise with gaussian distribution with $\sigma=10$ 


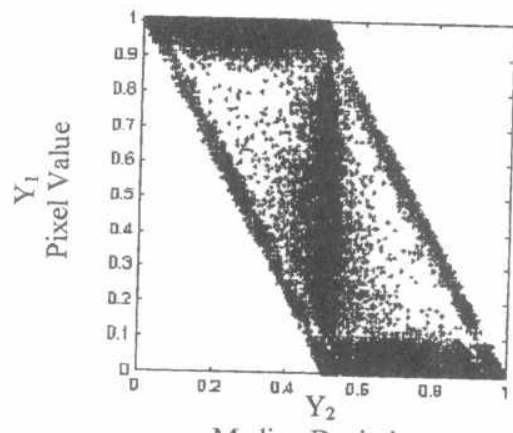

Median Deviation

Fig. 10(a) Input vectors

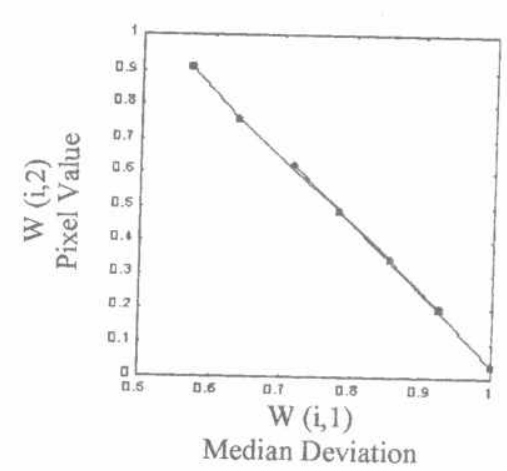

Fig. 10(b) Output clusters

Fig. 10 Noisy Lenna input vectors and output clusters of ANN applied to Lenna image corrupted by $60 \%$ impulse noise with gaussian distribution with $\sigma=10$

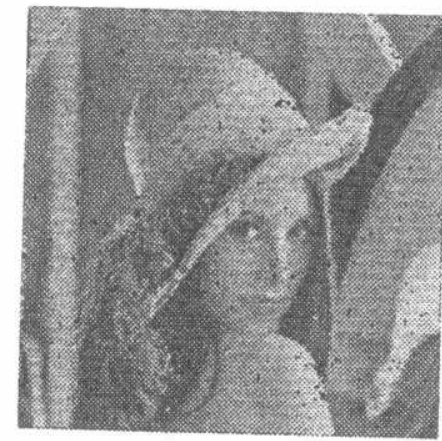

Fig. 11(a) NEAM filtered

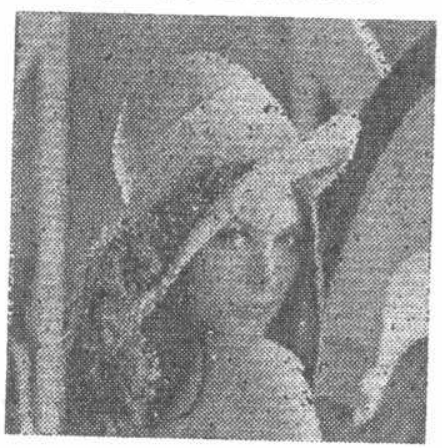

Fig. 11(c) NEN filtered

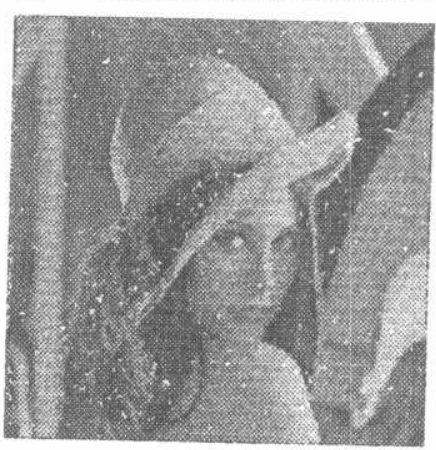

Fig. 11(b) NEM filtered

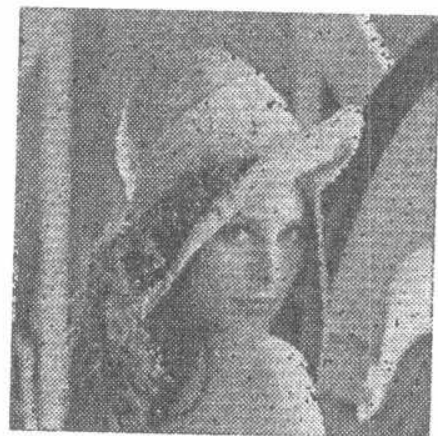

Fig. 11(d) NEMM filtered

Fig. 11 Noise-exclusive filtered images of Lenna image corrupted by $60 \%$ impulse noise with gaussian distribution with $\sigma=10$ 


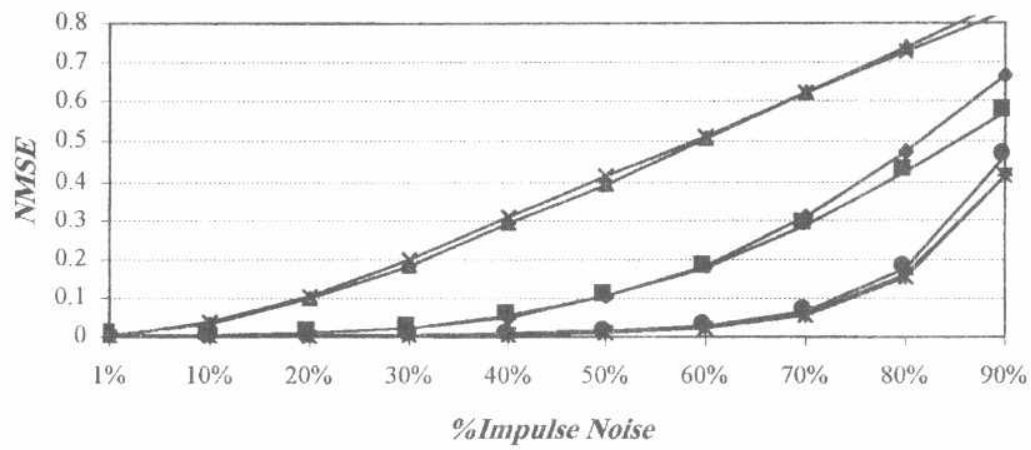

$\begin{array}{llll}\rightarrow \text { Median } & - \text { CWM } & \rightarrow \text { Max/Med } & * \text { Multistage } \\ \text { *-NEAM } & - \text { NEM } & \text {-NEN }\end{array}$

Fig. 12 The performance comparison of filters on Lenna image corrupted by different percentages of impulse noise with uniform distribution

$$
\text { with } \sigma=20
$$
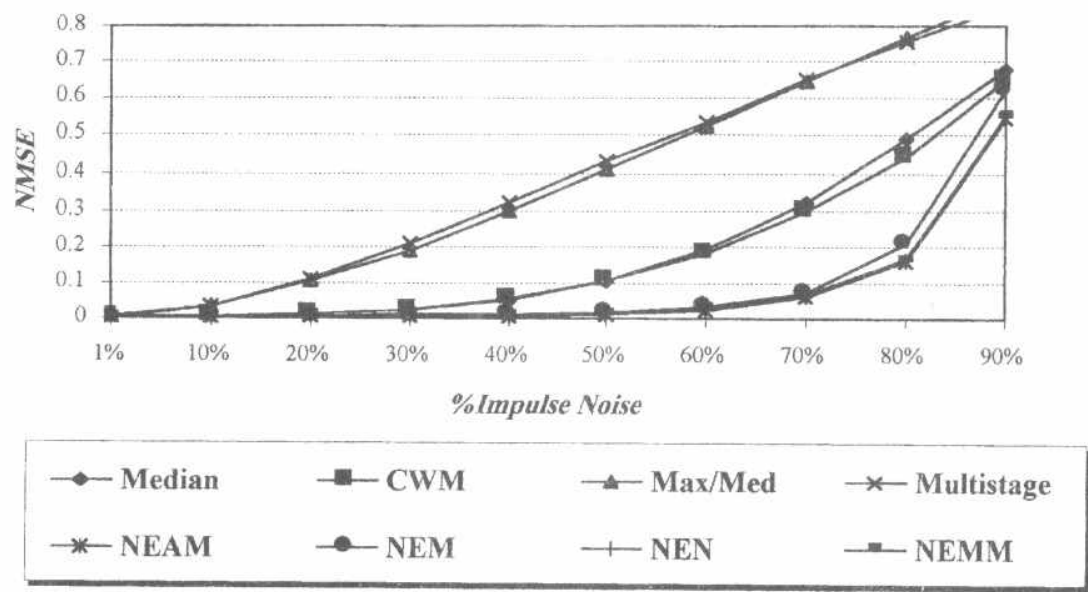

Fig. 13 The performance comparison of filters on Lenna image corrupted by different percentages of impulse noise with gaussian distribution

$$
\text { with } \sigma=10
$$




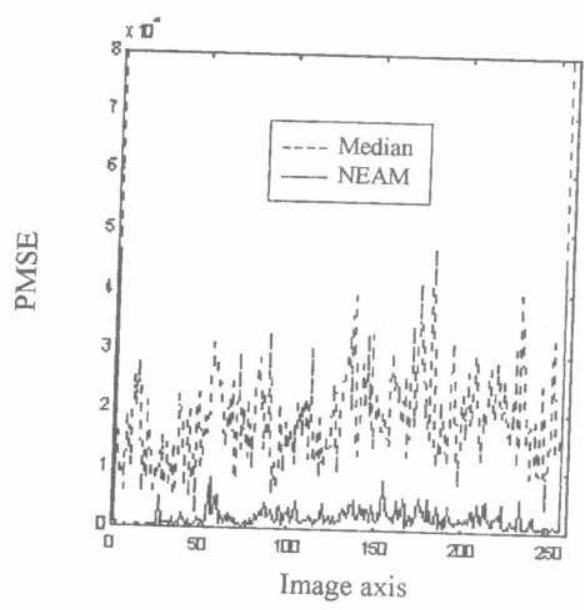

Fig. 14 The PMSE calculated for NEAM filter and Median filter applied on Lenna image corrupted by $40 \%$ impulse noise with uniform distribution with $\sigma=20$

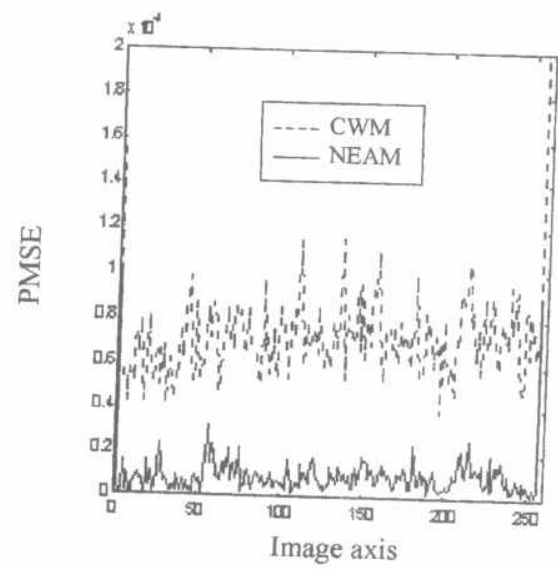

Fig. 15 The PMSE calculated for NEAM filter and CWM filter applied on Lenna image corrupted by $60 \%$ impulse noise with gaussian distribution with $\sigma=10$ 